\title{
ANÁLISE DAS RELAÇÕES ENTRE DESFLORESTAMENTOS E FOCOS DE CALOR. ESTUDO DE CASO NOS MUNICÍPIOS DE ALTAMIRA E SÃO FÉLIX DO XINGU, NO ESTADO DO PARÁ ${ }^{1}$
}

\author{
Luciano Lamper Martinez ${ }^{2}$, Nilton César Fiedler ${ }^{3}$ e Geraldo José Lucatelli ${ }^{4}$
}

\begin{abstract}
RESUMO - Este estudo foi desenvolvido em área-piloto da região conhecida como "Terra do Meio" localizada nos municípios de Altamira e São Félix do Xingu, no Estado do Pará, tendo como objetivo geral analisar as relações entre os desflorestamentos e os focos de calor no período de 1998 a 2002. Como objetivos específicos, o trabalho procurou verificar se existe relação entre o tamanho da área desflorestada e a incidência de focos de calor; e averiguar se os processos de obtenção e geoposicionamento das imagens NOAA interferem na sobreposição de focos de calor com polígonos de desflorestamento. Os dados foram analisados em um sistema de informações geográficas SIG. Para a determinação da relação entre as variáveis foram realizadas duas análises de regressão, uma linear e outra polinomial de $2^{\circ}$ grau. Obtidas as equações, foi realizado um teste de significância dos componentes linear e quadrático e calculado o coeficiente de determinação da escolha da equação que melhor se ajusta à distribuição dos dados. Verificou-se relação positiva entre os dados analisados. A relação foi maior quando foram sobrepostos focos de calor e incrementos de desflorestamento do mesmo período. Os resultados mostraramse mais consistentes nas maiores áreas de desflorestamento. Os componentes linear e quadrático das equações obtidas mostraram-se significantes utilizando o teste " $t$ ". Por serem mais simples, optou-se pelas equações obtidas pelo método de regressão linear, para representar a distribuição dos dados. O maior número de sobreposições entre os focos de calor e os desflorestamentos com área superior a 100 ha indicam que o tamanho do desflorestamento influencia diretamente a detecção de focos. Os processos de obtenção e geoposicionamento das imagens NOAA não interferem nessa sobreposição. Os resultados evidenciam que os produtos gerados pelos satélites NOAA são muito apropriados para detecção de focos de calor, porém imprecisos na localização e dimensionamento das áreas afetadas.
\end{abstract}

Palavras chave: Geoposicionamento, Amazônia e Terra do Meio.

\section{ANALYSIS OF THE RELATIONSHIP BETWEEN DEFORESTATION AND HOTSPOTS. CASE STUDY IN THE MUNICIPAL DISTRICTS OF ALTAMIRA AND SÃO FÉLIX DO XINGU IN THE STATE OF PARÁ}

\begin{abstract}
The present study was developed in a test field of an area known as "Terra do Meio". It is located in the municipal districts of Altamira and São Félix do Xingu, State of Pará. The general objective of this work was to analyze the relationship between deforestation and hotspots in the period from 1998 to 2002. The specific objectives were to verify whether there is any relationship between the size of the deforested area and the incidence of hotspots; as well as to find out whether the process of obtaining and geopositioning of NOAA images interferes in the overlapping of hotspots with polygons of deforestation. The data were analyzed in a geographical information system (S.I.G.). For determining the relation between the variables, two regression analyses were carried out: linear and $2^{\text {nd }}$ degree polynomial. Once the equations were obtained, a test of significance of the linear and quadratic components was accomplished. Then, the coefficient of determination
\end{abstract}

\footnotetext{
${ }^{1}$ Recebido em 16.02.2006 e aceito para publicação em 29.03.2007.

${ }^{2}$ Engenheiro Florestal, Polícia Federal - Boa Vista-RR. E-mail: <lucianolamper@ gmail.com>.

${ }^{3}$ Departamento de Engenharia Florestal, Centro de Ciências Agrárias da UFES, 29500-000 Alegre-ES. E-mail:<fiedler@pq.cnpq.br>.

${ }^{4}$ IBAMA-Sensoriamento Remoto-SCEN Trecho 2,Cx. Postal 09870, 70818-900 Brasília-DF. E-mail:<glucatelli@ sede.ibama.gov.br>.
} 
was calculated, in order to choose the best-fit equation to the data distribution. Positive relationship was found within the analyzed data. The relationship was greater when hotspots and increase in deforestation in the same period were overlapped. The largest deforestation areas showed more consistent results. The linear and quadratic components of the resulting equations were more significant with the use of " $t$ " test. The equations obtained by the method of linear regression were chosen to represent the data distribution, because they are considered simpler. The larger number of overlapping between the hotspots and deforestation in areas of more than 100 hectares suggests that the deforestation size directly influences fire detection. The obtaining and geopositioning of NOAA images do not interfere in this overlapping. The results suggest that the products generated by NOAA satellites are suitable for hotspot detection. However, they are imprecise when it comes to locating and dimensioning the affected areas.

Keywords: Geopositioning, Amazônia and Terra do Meio.

\section{INTRODUÇÃO}

A Amazônia brasileira representa $41 \%$ das florestas tropicais restantes no mundo (FAO, 1993) e exerce papel vital na manutenção da biodiversidade, no equilíbrio hidrológico e climático e no armazenamento terrestre de carbono (FEARNSIDE, 1999). Ocupando cerca de $59 \%$ do território nacional, abrange os Estados do Acre, Amapá, Amazonas, Maranhão, Mato Grosso, Pará, Rondônia, Roraima e Tocantins. Essa imensa área, superior a 5 milhões de $\mathrm{km}^{2}$, é suficiente para proporcionar variabilidade de condições ambientais responsável pela heterogeneidade espacial da região (KAMPEL et al., 2001).

Diversos estudos sobre o ecossistema amazônico têm contado que, nos últimos anos, alterações significativas, embora localizadas, nos fluxos de água, energia, carbono, e ciclagem de nutrientes e na composição da atmosfera foram causadas por desflorestamentos e queimadas (NETTO, 2002). A rápida conversão de extensas áreas de Floresta Tropical Amazônica em áreas agrícolas, principalmente pastagens, provoca alterações no balanço de carbono e no ciclo hidrológico, acúmulo de gases do efeito-estufa na atmosfera e a extinção de espécies.

Os levantamentos sobre as dinâmicas de ocupação humana na Amazônia baseados na análise de produtos de sensores ópticos tiveram início na década de 1970. Os levantamentos de áreas desflorestadas baseados nos sensores LANDSAT/MSS (TARDIN et al., 1980) e LANDSAT/TM (INPE, 1999, 2000 e 2002) tornaram possível o mapeamento da extensão dos danos e a estimativa das taxas de desflorestamento (ESCADA et al., 2001). As análises dos processos de ocupação humana na Amazônia, com base em produtos de sensores ópticos, permitem investigar a distribuição espacial dos desflorestamentos (TARDIN et al., 1980; ALVES et al., 1991) e identificar áreas de ocupação mais intensa (INPE, 1999, 2000; ALVES, 2001). O levantamento da cobertura florestal da Amazônia utilizando tecnologia de sensores remotos oferece meio único de monitorar a região a custos aceitáveis (ALVES, 2001).

Segundo Alves (2001), alguns estudos apresentam restrições relacionadas com os aspectos metodológicos, a não-disponibilidade de dados para algumas regiões, a cobertura de nuvens, o intervalo de tempo entre as análises, a desconsideração de erros de delineamento, o posicionamento e detecção das áreas desflorestadas e a não-geração de mapas com qualidade e formato adequados para a disseminação e publicação. Análises recentes utilizando técnicas de processamento digital de dados orbitais eliminaram o problema de distorções geométricas e a conseqüente falta de ajuste dos dados quando se trabalha com imagens de épocas distintas. Trata-se das análises multitemporais (SHIMABUKURO et al., 1999).

As queimadas estão amplamente inseridas no processo produtivo da Amazônia e constituem um dos elementos que impulsionam a expansão agrícola na região. Considerado um método barato de preparar a terra para o plantio de culturas e para a limpeza e renovação de pastagens, o fogo queima as árvores derrubadas, produzindo cinzas ricas em nutrientes que fertilizam o solo e, no curto prazo, aumentam a sua produtividade. $\mathrm{O}$ fogo também estimula o crescimento das gramíneas forrageiras e elimina as plantas invasoras lenhosas das pastagens. Sem o fogo, o investimento na compra de máquinas pesadas e o elevado tempo gasto para eliminar as ervas daninhas seriam inevitáveis (NEPSTAD et al., 2001). 
As imagens dos satélites NOAA são utilizadas, entre outras coisas, para monitorar a ocorrência de focos de calor na superfície terrestre. Os processamentos realizados pela Divisão de Satélites Ambientais do Centro de Previsão do Tempo e Estudos Climáticos (DSA/ CPTEC) do INPE consistem no geoposicionamento (localização) e discretização (classificação) dos focos de calor. A quantidade de nuvens, os movimentos dos satélites, o tempo de transmissão, o posicionamento em relação à área central imageada (efeito de borda) e os sistemas de classificação utilizados podem diminuir não só a precisão da localização dos focos, como também o número e a confiabilidade dos focos detectados.

O objetivo geral deste estudo foi analisar as relações entre os desflorestamentos e os focos de calor, no período de 1998 a 2002, em área-piloto da região conhecida como "Terra do Meio", localizada nos Municípios de Altamira e São Félix do Xingu, no Estado do Pará, a fim de contribuir para a elaboração de estratégias que auxiliem o controle e mitigação dos desflorestamentos e queimadas na Amazônia. Seus objetivos específicos foram:

1. Verificar se existe relação entre o tamanho da área desflorestada e a incidência de focos de calor.

2. Averiguar se os processos de obtenção e geoposicionamento dos focos de calor interferem na sobreposição das áreas desflorestadas.

\section{MATERIAL E MÉTODOS}

\section{1. Área de Estudo}

Esta pesquisa teve como área de estudo parte da região conhecida como "Terra do Meio", localizada entre as coordenadas 5 $5^{\circ} 35^{\prime} 24^{\prime \prime} \mathrm{S}, 5^{\circ} 18^{\prime} \mathrm{W}$ e $7^{\circ} 10^{\prime} 48^{\prime}$ ' $\mathrm{S}$, $52^{\circ} 22^{\prime} 48^{\prime \prime} \mathrm{W}$, nos Municípios de Altamira e São Félix do Xingu, no Estado do Pará, com área total de $37.412,9815 \mathrm{~km}^{2}$ (Figura 1).

\subsection{Material}

Para a execução deste estudo foram utilizados os seguintes materiais:

- Imagens de satélite LANDSAT/TM, obtidas no Centro de Sensoriamento Remoto do IBAMA, das seguintes órbitas/ponto:

o 225-64 - passagens de 30.06.1998, 03.07.1999, 06.08.2000, 17.08.2001 e 03.07.2002. o 225-65 - passagens de 29.05.1998, 04.08.1999, 06.08.2000, 14.06.2001 e 20.08.2002.

o 226-64 - passagens de 09.09.1998, 10.07.1999, 04.07.2000, 08.08.2001 e 11.08.2002.

o 226-65 - passagens de 24.08.1998, 10.07.1999, 17.05.2000, 08.08.2001 e 26.07.2002.

- Focos de calor dos anos de 1998, 1999, 2000, 2001 e 2002, acumulados por período e obtidos na Gerência de Monitoramento do PROARCO/IBAMA.

- Computador AMD Athlon XP, processador 1800+ MHz, 40 Gbytes de disco, 512 Mbytes de memória RAM.

- Software de geoprocessamento ArcView versão 3.2 e extensão para processamento de imagens Image Analysis versão 1.0.

- Software para tratamento de imagens Erdas Imagine versão 8.3.1.

- Dois aparelhos para Posicionamento Global - GPS, da marca Garmin, modelo 12.

- Planilha Gráfica/Estatística Microsoft Excel 2000 e SPSS versão 8.0.

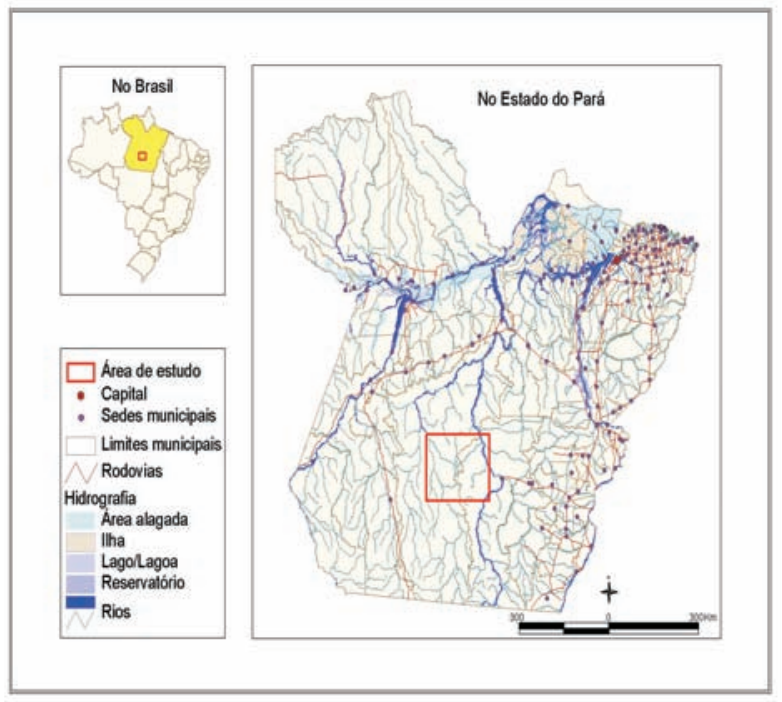

Fonte: Figura elaborada pelo autor. / Font: Figure by the author.

Figura 1 - Localização da área de estudo.

Figure 1 -Location of study area.

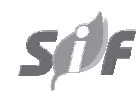

R. Árvore, Viçosa-MG, v.31, n.4, p.695-702, 2007 


\subsection{Base de Dados}

Neste estudo foram analisados o desflorestamento acumulado até 1998 e os incrementos de 1999, 2000, 2001 e 2002, bem como o número total de focos de calor nos anos de 1998, 1999, 2000, 2001 e 2002. As imagens de 2002 foram georreferenciadas em escala 1:100.000, e ajustaram-se perfeitamente às estradas e vias de acesso mapeadas por terra, utilizando-se dois aparelhos de GPS. As imagens do ano de 2002 serviram de referência para o georreferenciamento das demais imagens, executado nos softwares Erdas Imagine e ArcView e utilizando a extensão Image Analyses.

Os dados de desflorestamentos utilizados neste estudo foram extraídos das imagens LANDSAT/TM, utilizando-se a metodologia desenvolvida no Centro de Sensoriamento Remoto - CSR do IBAMA e aplicada por Teixeira et al., (1999) em estudo sobre a dinâmica do desflorestamento na Amazônia.

As informações sobre os focos de calor foram fornecidas pelo PROARCO/IBAMA. Utilizou-se o algoritmo Clip themes do software ArcView para gerar, a partir dos arquivos de focos de calor acumulado de todo o país, cinco novos arquivos, um para cada período (1998, 1999, 2000, 2001 e 2002), para a área de estudo.

Para correlacionar os focos de calor e os incrementos de desflorestamento e testar se erros associados aos processos de aquisição e geoposicionamento das imagens NOAA interferem na correlação entre focos de calor e desflorestamento, foram gerados dois buffers, um com três e outro com $6 \mathrm{~km}$ de raio em torno das coordenadas pontuais dos focos de calor. Os dois buffers foram gerados utilizando-se o algoritmo para criação de buffers do software ArcView.

\subsection{Métodos de Análise}

A fim de verificar a correlação entre o desflorestamento e os focos de calor, os dados foram analisados em um sistema de informações geográficas, gerando 27 combinações diferentes.

O desflorestamento acumulado até 1998, os incrementos de desflorestamento dos anos de 1999, 2000, 2001 e 2002 e os totais acumulados em cada período foram cruzados com os respectivos arquivos dos focos de calor e com os buffers de 3 e $6 \mathrm{~km}$ de raio, criados em torno das coordenadas pontuais dos focos.

R. Árvore, Viçosa-MG, v.31, n.4, p.695-702, 2007
Buscando identificar padrões e alterações sutis nas dinâmicas do desflorestamento que possam explicar as variações dos resultados, os incrementos de desflorestamento foram divididos em quatro classes, por tamanho de área:
i. 0 a 100 hectares.
ii. 100 a 500 hectares.
iii. 500 a 1.000 hectares.
iv. 1.000 a 5.000 hectares.

\subsection{Cruzamento dos Dados}

Para realização das combinações entre os focos de calor e os desflorestamentos foram utilizados três algoritmos (Intersect Themes, Summarize e Join Tables) do software ArcView, da seguinte maneira:

1. Interseção dos arquivos de focos de calor gerados para a área de estudo e arquivos de desflorestamentos para identificação do número de focos que se sobrepõem às áreas desflorestadas. Foi gerado um resultado intermediário, na forma de tema do ArcView (Interseção Focos X Desflorestamentos).

2. Contabilização do número de focos de calor associado a cada polígono de desflorestamento desse tema intermediário, gerando uma tabela resumida do número de focos de calor associado a cada polígono de desflorestamento.

3. Junção da tabela resumida com a tabela dos desflorestamentos para totalização das informações do número total de focos de calor por polígono de desflorestamento e identificação daqueles polígonos que não foram sobrepostos por focos de calor.

4. Exportação das informações sobre o número total de focos de calor por polígono de desflorestamento no formato DBF e análise das planilhas gráfica/ estatística Microsoft Excel 2000 e SPSS 8.0.

\subsection{Análise Estatística}

De acordo com a hipótese do trabalho, o número de focos foi definido como a função do desflorestamento, ou seja:

$$
\begin{aligned}
& y=f(x) \\
& y=f(\text { desflorestamento })
\end{aligned}
$$

em que: 
$\mathrm{x}=$ variável independente (desflorestamento); e

$\mathrm{y}$ = variável dependente (número de focos de calor).

As combinações entre focos de calor e desflorestamentos foram testadas utilizando-se duas regressões, uma linear simples e outra polinomial de segundo grau, para verificar qual se ajustava melhor à distribuição dos dados. Em cada uma das combinações entre desflorestamentos e focos de calor detectados na área de estudo para escolha da equação de regressão, consideraram-se a análise de variância da regressão, o coeficiente de determinação e o teste de " $t$ " (GOMES, 1987; HOFFMANN e VIEIRA, 1983).

\section{RESULTADOS E DISCUSSÃO}

Os dados sobre o desflorestamento ocorrido no período analisado indicaram crescimento em área com comportamento exponencial (Figura 2).

O início da intensificação dos processos de ocupação mostrou-se evidente com o incremento de desflorestamento registrado em 2000. Segundo Moran et al. (1996), o crescimento econômico associado ao controle da inflação proporcionou novo aumento nas taxas de desflorestamento.

No período de 1999 a 2002, a área total desflorestada aumentou mais de quatro vezes. A conversão de grandes áreas de florestas em pastagens refletiu o avanço e consolidação da fronteira agrícola; nesse caso, a pecuária. O maior valor alcançado pela terra desmatada, por ser considerada beneficiada, desencadeou um mercado de terras que favoreceu o desflorestamento (Moran et al., 1996).



Figura 2 - Evolução do desflorestamento na área de estudo no período de 1998 a 2002.

Figure 2 -Evolution of deforestation in the study area from 1998 to 2002
A quantidade de focos de calor detectados pelo satélite NOAA nos períodos analisados, assim como os incrementos de desflorestamento, apresentou comportamento exponencial (Figura 3).

Esse resultado evidencia indícios da relação positiva entre o desflorestamento e o número de focos de calor ocorridos na área de estudo no período analisado. $\mathrm{O}$ aumento proporcional no número de focos de calor registrados pelos satélites NOAA em relação aos incrementos anuais de desflorestamento tem ligação com a relação positiva entre essas variáveis.

A significância dos componentes linear e quadrático das equações de regressão obtidas por meio do teste " $t$ " indicou que todas as equações lineares são significativas para os dados analisados, à exceção da combinação realizada entre o incremento de desflorestamento e os focos de calor ocorridos em 1999. A lenta dinâmica de alteração da cobertura florestal registrada nesse período justifica esse resultado, pois o grande número de áreas desflorestadas encontrado nas classes de menores tamanhos aumenta a probabilidade de erro de detecção das imagens dos satélites NOAA que, até então, eram processadas em sistemas de oito bits. A evolução dos sistemas de processamento das imagens NOAA, de 8 para 10 bits, ocorrida em 2001, proporcionou aumento de cerca de $30 \%$ no número de focos de calor confiáveis, e diminuiu o número de prováveis focos de calor descartados.

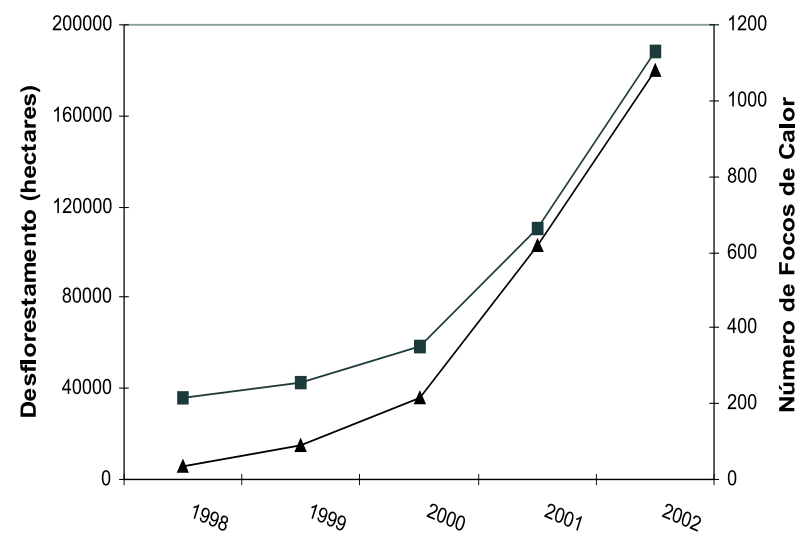

Figura 3 - Evolução da área desflorestada e do número de focos de calor detectados na área de estudo, no período de 1998 a 2002.

Figure 3 - Evolution of deforested area and number of hotspots detected in the study area from 1998 to 2002.

R. Árvore, Viçosa-MG, v.31, n.4, p.695-702, 2007 
Os coeficientes de determinação obtidos a partir da regressão polinomial foram maiores que os conseguidos a partir da regressão linear simples. As curvas traçadas por equações de segundo grau indicaram melhor ajuste que as retas obtidas utilizando o método de regressão linear simples.

Pode-se observar aumento progressivo dos coeficientes de determinação no período de 1998 a 2002, com exceção de 1999, justificado pelas diferenças na distribuição das áreas desflorestadas em classes por tamanho. Os desflorestamentos de menores áreas identificados em 1999 apresentaram menor relação com os focos de calor detectados na área de estudo.

No intervalo de tempo entre os anos de 1999 e 2002, pode-se observar maior relação entre os incrementos de desflorestamento e os focos de calor ocorridos nos mesmos períodos, ou seja, incrementos de desflorestamento com focos de calor do mesmo ano. As relações observadas entre o desflorestamento acumulado e os focos de calor de cada ano foram menores. Esses resultados evidenciam que na área de estudo e no período analisado a relação entre os focos de calor e os incrementos de desflorestamentos foi maior. Esses focos detectados estão associados às atividades de limpeza de restos de exploração de floresta nativa para implantação de pastagens.

Tratando-se de uma área com ocupação recente, os focos de calor detectados não estão associados às atividades de limpeza e renovação de pastagens como em outras regiões de ocupação mais antiga. Os maiores coeficientes de determinação encontrados nas combinações entre os desflorestamentos e os focos de calor dos mesmos períodos na área de estudos confirmam esses resultados.

As análises realizadas com os buffers de 3 e 6 km apresentaram coeficientes de determinação menores que aqueles obtidos a partir das coordenadas pontuais dos focos de calor, tanto por meio do método de regressão polinomial quanto pela regressão linear simples. Os decréscimos nos coeficientes de determinação verificados indicaram que o aumento da área de contato entre os desflorestamentos e focos de calor obtidos com a utilização dos buffers, ao contrário do esperado, não fez com que os coeficientes de determinação fossem maiores. A coordenada pontual do foco de calor mostrouse a melhor unidade de relação entre os incrementos de desflorestamentos e os focos de calor.

R. Árvore, Viçosa-MG, v.31, n.4, p.695-702, 2007
O aumento dos coeficientes de determinação no período de 1998 a 2002 está relacionado ao incremento no número de áreas desflorestadas registrado nas classes de maiores tamanhos e também à melhoria nos processos de classificação e geoposicionamento das imagens NOAA, evolução do sistema de 8 para 10 bits.

O aumento proporcional não só do número de áreas desflorestadas detectadas em imagens LANDSAT/TM, como também do número de focos de calor registrados anualmente pelos satélites NOAA, está relacionado ao aumento do número de sobreposições entre focos de calor e desflorestamentos, verificado na área de estudo no período entre os anos de 1998 a 2002.

O reduzido número de focos de calor detectado pelo satélite NOAA em 1998 está atribuído à lenta alteração da cobertura florestal que ocorria na área de estudo até esse período. A ausência de incremento de novas áreas desflorestadas nas classes de maiores tamanhos justifica a detecção reduzida do número de focos de calor, visto que a queima para limpeza de pequenas pastagens e áreas agrícolas não é detectada pelos sensores dos satélites NOAA. Considerando que os desflorestamentos detectados em 1999 ocorreram principalmente na primeira classe de tamanho (até 100 ha), pode-se extrapolar que isso aconteceu em 1998, ano-base. Dos oito focos de calor que sobrepuseram o desflorestamento acumulado até 1998, seis foram na classe com as maiores áreas.

A discrepância observada nos valores dos coeficientes de determinação obtidos em 1999 é explicada pela pequena sobreposição ocorrida entre focos de calor e os incrementos de desflorestamento identificados na área de estudo nesse ano. A maior ocorrência de desflorestamentos na primeira classe de tamanho justifica as baixas relações entre os focos de calor e o desflorestamentos obtidos nos anos de 1998 e 1999.

Os maiores coeficientes de determinação encontrados na combinação entre os desflorestamentos acumulados e os focos de calor detectados na área de estudo, em 1999, estão relacionados à limpeza e renovação de antigas pastagens, pois os valores obtidos nas combinações entre o incremento de desflorestamento e os focos de calor registrados, ao contrário dos outros períodos analisados, foram menores.

A relação entre focos de calor e desflorestamentos na área de estudo foi maior nas classes de tamanho 
com as maiores áreas, pois nas classes com áreas menores desflorestadas registraram-se menores números de sobreposições com os focos de calor, enquanto nas classes com maiores áreas foi registrado maior número de sobreposições. O número de focos de calor registrado por polígono de desflorestamento está relacionado com o tamanho das áreas desflorestadas.

O maior número de sobreposições entre os focos de calor e os desflorestamentos com área superior a 100 ha registrados na área de estudo indicam que o tamanho do desflorestamento influencia diretamente a detecção de focos e, conseqüientemente, as relações entre as variáveis desflorestamento e focos de calor.

A variação do número de sobreposições de focos de calor e desflorestamento também está associada à resolução espacial das imagens captadas pelo sensor AVHRR. Os dados obtidos nas regiões das bordas são menos precisos que os da região central das imagens NOAA. Mesmo que alguns focos sejam detectados na região de bordas das imagens, há maior probabilidade de localização incorreta desses dados.

\section{CONCLUSÕES}

As análises dos resultados encontrados neste estudo permitem a conclusão de que existe relação positiva entre focos de calor e desflorestamentos, e essa relação foi maior quando foram sobrepostos focos de calor e incrementos de desflorestamento do mesmo período. A maior parte das ocorrências de focos de calor na área de estudo estava associada à conversão de áreas de floresta em pastagens.

Os processos de geoposicionamento das imagens NOAA não interferem na sobreposição de focos de calor com polígonos de desflorestamento. O tamanho do desflorestamento influencia diretamente a detecção de focos e, conseqüentemente, a relação entre as variáveis focos de calor e desflorestamento.

A resolução espacial do sensor AVHRR é o principal limitante da localização precisa dos focos de calor. A redução na resolução no sentido centro/borda da imagem influencia a localização. Os produtos gerados pelos satélites NOAA são muito interessantes para detecção de focos de calor, porém imprecisos na localização e dimensionamento das áreas afetadas. Essa imprecisão quanto à localização é maior nas áreas menores. Os resultados mostraram-se mais consistentes nas maiores áreas de desflorestamento.
Os componentes linear e quadrático das equações obtidas mostraram-se significantes utilizando o teste " $t$ " em quase todas as combinações. Por serem mais simples que as equações obtidas com a regressão polinomial de $2^{\circ}$ grau, optou-se pelas equações obtidas pelo método de regressão linear para representar a distribuição dos dados.

A conversão de grandes áreas de florestas em pastagens, como a verificada na área de estudo, acentua a necessidade de elaboração de políticas que orientem a utilização adequada desses recursos com vistas ao desenvolvimento sustentável da região.

A ausência total do Estado permitiu que proprietários já estabelecidos na margem direita do rio Xingu e outros oriundos, principalmente, do Estado do Tocantins definissem as formas de ocupação dessa nova área de expansão. Tais processos desordenados de ocupação, se não mitigados imediatamente, impossibilitarão a utilização sustentável dos recursos naturais na região estudada.

Considerando-se o alto custo de obtenção de imagens LANDSAT, o problema de calibração do satélite LANDSAT 7 e o intervalo de tempo para obtenção desses produtos, o uso de imagens NOAA como ferramenta de monitoramento e controle das dinâmicas de alteração da cobertura florestal, para direcionamento de ações de ordenamento e gestão territorial, é altamente recomendável.

\section{REFERÊNCIAS}

ALVES, D.S. O processo de desflorestamento na Amazônia. Parcerias Estratégicas, n.6, p.259-266. 2001.

ESCADA, M. I. S.; ALVES, D. S. Mudanças de uso e cobertura do solo na Amazônia: impactos sócio-ambientais na ocupação de regiões de fronteira agrícola.. São José dos Campos: Instituto de Pesquisas Espaciais, 2001. (Relatório Técnico Parcial)

FAO. Forest Resources Assessment 1990: tropical countries. Rome: FAO, 1993. 61p. (FAO Forestry Paper 112).

R. Árvore, Viçosa-MG, v.31, n.4, p.695-702, 2007 
FEARNSIDE, P. M. Biodiversity as an environmental service in Brazil's Amazonian forests: risks, value and conservation. Environmental Conservation, v.26, p.305-321, 1999.

GOMES, F. P. Curso de estatística experimental. 12.ed. Piracicaba: Nobel, ESALQ, 1987. 468p.

HOFFMANN, R.; VIEIRA, S. Análise de regressão: uma introdução à econometria. 2.ed. São Paulo: Hucitec, 1983. 379p.

INSTITUTO NACIONAL DE PESQUISAS ESPACIAIS - INPE. Monitoramento da floresta amazônica por satélite 19971998. São José dos Campos: 1999.

INSTITUTO NACIONAL DE PESQUISAS ESPACIAIS - INPE. Monitoramento da Floresta Amazônica Brasileira por Satélite 1999-2000. São José dos Campos: 2000.

INSTITUTO NACIONAL DE PESQUISAS ESPACIAIS - INPE. Monitoramento da Floresta Amazonica Brasileira por Satélite 2000-2001. São José dos Campos: 2002.
KAMPEL, S. A.; CÂMARA, G.; MONTEIRO, A. $M$. V. Configurações espaciais do processo de desflorestamento da Amazônia. São José dos Campos: Instituto de Pesquisas Espaciais, 2001. (Relatório Técnico)

MORAN, E. F. et al. Restoration of vegetation cover in the eastern Amazon. Ecological Economics, v.18, p.41-54, 1996.

NEPSTAD, D. et al. Road paving, fire regime feedbacks, and the future of Amazon Forests. Forest Ecology \& Management, v.154, p.395-407, 2001.

NETTO, P. E. A. Mudanças de uso de solo na Amazônia: implicações climáticas e na ciclagem de carbono. Manaus: Instituto Nacional de Pesquisas da Amazônia-INPA, 2002. (Reunião Científica)

SHIMABUKURO, Y. E. et al. Levantamento de áreas desflorestadas na Amazônia através de processamento digital de imagens orbitais. Floresta e Ambiente, v.6, n.1, p.38-44, 1999.

TARDIN, A. T. et al. Subprojeto desmatamento São José dos Campos: Convênio IBDF/CNPq/INPE, 1980.

TEIXEIRA, C. V. et al. Geoprocessamento e a fiscalização do desmatamento. In: GIS BRASIL 1999. Salvador. Anais... Salvador: 1999. CD-ROM. 